\title{
Geometric and constitutive dependence of Maxwell's velocity slip boundary condition
}

\author{
D. A. Lockerby ${ }^{1}$, J. M. Reese ${ }^{2}$, R. W. Barber ${ }^{3}$ and D. R. Emerson ${ }^{3}$ \\ ${ }^{I}$ Department of Mechanical Engineering, King's College London, London WC2R 2LS, UK \\ ${ }^{2}$ Department of Mechanical Engineering, University of Strathclyde, Glasgow G1 1 XJ, UK \\ ${ }^{3}$ Centre for Microfluidics, CCLRC Daresbury Laboratory, Warrington, Cheshire WA4 4AD, UK
}

\begin{abstract}
The general form of Maxwell's velocity slip boundary condition for rarefied gas flows depends on both the geometry of the surface and the constitutive relations used to relate the viscous stress to rate of strain. The dependence on geometry is often overlooked in current rarefied flow calculations, and the generality of the constitutive dependence means the condition can also be usefully applied in regions where the Navier-Stokes equations fail, e.g. rarefied flows close to surfaces. In this paper we give examples illustrating the importance of both these dependencies and show, therefore, that implementing the general Maxwell condition produces substantially different results to conventional implementations of the condition. Finally, we also investigate a common numerical instability associated with Maxwell's boundary condition, and propose an implicit solution method to overcome the problem.
\end{abstract}

\section{MAXWELL'S VELOCITY SLIP BOUNDARY CONDITION}

In 1879, James Clerk Maxwell published a paper on the viscous stresses arising in rarefied gases [1]. At the time, a reviewer commented that it also might be useful if Maxwell could use his theoretical findings to derive a velocity boundary condition for rarefied gas flows at solid surfaces. Consequently, in an appendix to the paper, Maxwell proposed his now-famous velocity slip boundary condition. This boundary condition was successful in predicting two prior experimental observations: (a) that a rarefied gas could slide over a surface, and (b) that inequalities in temperature could give rise to a force tending to make the gas slide over a surface from colder to hotter regions (which had been discovered by Reynolds, and was known as "thermal transpiration" - now more commonly known as "thermal creep"). What has subsequently been overlooked by many current researchers is the general form of the slip expression derived by Maxwell, and this has some substantial consequences for modern simulations of, e.g., hypersonic aerodynamics and gas flows in microsystems.

Maxwell related the tangential gas velocity slip, $\vec{u}_{\text {slip }}$ to the tangential shear stress, $\vec{\tau}$, and heat flux, $\vec{q}$. Writing his expression in vector form so that it can be easily applied to flows over three-dimensional surfaces, we have:

$$
\vec{u}_{s l i p}=-A_{1} \frac{(2-\sigma)}{\sigma \mu} \lambda \vec{\tau}-\frac{3}{4} \frac{\operatorname{Pr}(\gamma-1)}{\gamma p} \vec{q}
$$

where $\vec{\tau}=\left(\vec{i}_{n} \cdot \boldsymbol{\Pi}\right) \cdot\left(\mathbf{1}-\vec{i}_{n} \vec{i}_{n}\right), \vec{q}=\vec{Q} \cdot\left(\mathbf{1}-\vec{i}_{n} \vec{i}_{n}\right)$, an arrow denotes a vector quantity, $\sigma$ is the momentum accommodation coefficient (equal to one for surfaces that reflect all incident molecules diffusely, and zero for purely specular reflection), $\mu$ is the gas viscosity at the wall, $\lambda$ is the molecular mean free path at the wall, Pr is the Prandtl number, $\gamma$ is the specific heat ratio, $p$ is the gas pressure at the wall, $\vec{i}_{n}$ is a unit vector normal and away from the wall, $\Pi$ is the stress tensor at the wall, $\mathbf{1}$ is the identity tensor and $\vec{Q}$ is the heat flux vector at the wall. Maxwell proposed a value for the slip coefficient, $A_{1}=1$, although a recent analytical solution of the linearized Boltzmann equation at a planar surface [2] estimates the "micro-slip" (the actual velocity slip at the wall) requires $A_{1}=\sqrt{ }(2 / \pi) \approx 0.8$. In [2], a value of $A_{1}=1.146$ is also proposed when the Navier-Stokes equations are used: this represents an additional "fictitious" velocity slip to accommodate for the Knudsen-layer structure that is not captured by linear constitutive relations [3].

CP762, Rarefied Gas Dynamics: $24^{\text {th }}$ International Symposium, edited by M. Capitelli (C) 2005 American Institute of Physics 0-7354-0247-7/05/\$22.50 


\section{Report Documentation Page}

Form Approved

OMB No. 0704-0188

Public reporting burden for the collection of information is estimated to average 1 hour per response, including the time for reviewing instructions, searching existing data sources, gathering and maintaining the data needed, and completing and reviewing the collection of information. Send comments regarding this burden estimate or any other aspect of this collection of information,

including suggestions for reducing this burden, to Washington Headquarters Services, Directorate for Information Operations and Reports, 1215 Jefferson Davis Highway, Suite 1204, Arlington

VA 22202-4302. Respondents should be aware that notwithstanding any other provision of law, no person shall be subject to a penalty for failing to comply with a collection of information if it

does not display a currently valid OMB control number.

\begin{tabular}{|c|c|}
\hline $\begin{array}{l}\text { 1. REPORT DATE } \\
\mathbf{1 3} \text { JUL } 2005\end{array}$ & $\begin{array}{l}\text { 3. DATES COVERED } \\
\text { - }\end{array}$ \\
\hline \multirow{3}{*}{$\begin{array}{l}\text { 4. TITLE AND SUBTITLE } \\
\text { Geometric and constitutive dependence of Maxwells velocity slip } \\
\text { boundary condition }\end{array}$} & 5a. CONTRACT NUMBER \\
\hline & 5b. GRANT NUMBER \\
\hline & 5c. PROGRAM ELEMENT NUMBER \\
\hline \multirow[t]{3}{*}{ 6. AUTHOR(S) } & 5d. PROJECT NUMBER \\
\hline & 5e. TASK NUMBER \\
\hline & 5f. WORK UNIT NUMBER \\
\hline $\begin{array}{l}\text { 7. PERFORMING ORGANIZATION NAME(S) AND ADDRESS(ES) } \\
\text { Department of Mechanical Engineering, Kings College London, London } \\
\text { WC2R 2LS, UK }\end{array}$ & $\begin{array}{l}\text { 8. PERFORMING ORGANIZATION } \\
\text { REPORT NUMBER }\end{array}$ \\
\hline \multirow[t]{2}{*}{ 9. SPONSORING/MONITORING AGENCY NAME(S) AND ADDRESS(ES) } & 10. SPONSOR/MONITOR'S ACRONYM(S) \\
\hline & $\begin{array}{l}\text { 11. SPONSOR/MONITOR'S REPORT } \\
\text { NUMBER(S) }\end{array}$ \\
\hline
\end{tabular}

12. DISTRIBUTION/AVAILABILITY STATEMENT

Approved for public release, distribution unlimited

13. SUPPLEMENTARY NOTES

See also ADM001792, International Symposium on Rarefied Gas Dynamics (24th) Held in Monopoli (Bari), Italy on 10-16 July 2004.

14. ABSTRACT

15. SUBJECT TERMS

16. SECURITY CLASSIFICATION OF:

a. REPORT unclassified

\section{b. ABSTRACT}

unclassified
17. LIMITATION OF ABSTRACT

$\mathbf{U U}$
18. NUMBER

OF PAGES

6 19a. NAME OF

RESPONSIBLE PERSON unclassified 
If the Navier-Stokes constitutive relations for stress and heat flux are substituted into Equation (1), expressions for velocity slip in terms of flow gradients can be obtained. In his original paper, Maxwell used a one-dimensional expression for the shear stress (appropriate for the typical case he was interested in) which made his final result generally applicable only to non-rotating planar walls (i.e. where the streamwise variation in wall-normal velocity is negligible). In scalar form, Maxwell gave:

$$
u_{s}=\frac{(2-\sigma)}{\sigma} \lambda \frac{\partial u_{x}}{\partial n}-\frac{3}{4} \frac{\mu}{\rho T} \frac{\partial T}{\partial x}
$$

where $n$ is the coordinate normal to the wall, $x$ is the coordinate tangential to the wall, $u_{x}$ is the $x$-component of the gas velocity, $u_{s}$ is the $x$-component of slip velocity, and $\rho, T$ are the density and temperature of the gas at the wall, respectively.

It is because of its relative simplicity, compared to Equation (1), that Equation (2) is remembered as Maxwell's main theoretical result. However, for most surface geometries of practical interest, having curvature and/or rotational motion, it is inapplicable because it neglects that the velocity normal to the wall can vary in the streamwise direction. Therefore a more complete expression for the tangential shear stress is required in Equation (1). If calculations straightforwardly applying Equation (2) are performed, they are likely to miss important features of the rarefied flow behavior.

\section{GEOMETRIC DEPENDENCE IN MAXWELL'S GENERAL FORMULATION}

If the full Navier-Stokes description of the stress tensor is adopted, for a wall in two dimensions, Equation (1) reduces to

$$
u_{s}=\frac{(2-\sigma)}{\sigma} \lambda\left(\frac{\partial u_{x}}{\partial n}+\frac{\partial u_{n}}{\partial x}\right)-\frac{3}{4} \frac{\mu}{\rho T} \frac{\partial T}{\partial x}
$$

where $u_{s}$ is the slip component tangential to the wall, and $u_{n}, u_{x}$ are the gas velocities normal and tangential to the wall, respectively.

The additional term that features in Equation (3) but not in Equation (2) can have a significant influence on the overall velocity slip. For example, when there is rotational wall motion (i.e. wall motion in a direction normal to the surface with a velocity that varies in the tangential direction) then even for flat surfaces (e.g. a deflecting flap) there will be a finite tangential velocity slip. For stationary walls, surface curvature will also give rise to a contribution from the additional term.

Although the misapplication of Equation (2) to general geometries is widespread, it is not universal, and there are instances where curved boundaries have been treated appropriately [4, 5]. Einzel, Panzer and Liu [6] derived a boundary condition similar to Equation (3) for surfaces with curvature. However, their boundary condition was formulated such that slip due to surface normal motion could not be accommodated. Also, it did not include the contribution of thermal creep to velocity slip, nor was the relationship to Maxwell's general equation realized. Two examples will show the importance of maintaining the geometric dependence that the general form of Maxwell's formulation incorporates.

\section{Cylindrical Couette Flow}

Recent analytical and molecular dynamics studies [5-7] suggest that the velocity profile in a rarefied cylindrical Couette flow can become inverted. In the case of a stationary outer cylinder and rotating inner cylinder, 'inverted' means that the radial velocity of the gas becomes greater further away from the moving centre.

We have performed a simple isothermal calculation using a finite difference discretization of the Navier-Stokes equations to examine the influence of various boundary conditions on the velocity profile. The inner and outer cylinders have radii of $3 \lambda$ and $5 \lambda$, respectively, and the former has a tangential velocity approximately a third of the speed of sound. The gas is argon at STP conditions and the accommodation coefficient, $\sigma$, is 0.1 . Figure 1 shows a comparison of the velocity profiles (non-dimensionalized by the tangential velocity of the inner cylinder) predicted using the standard no slip condition, the conventional slip condition (Equation 2), Maxwell's general slip condition 
(Equation 1), DSMC (direct simulation Monte Carlo) molecular dynamics [7], and the analytical method of Einzel, Panzer and Liu [6].

The DSMC method (being a statistical molecular dynamics simulation) is often used as an independent numerical test in the absence of experimental data [8]. That DSMC predicts an inverted velocity profile is strong corroborative evidence that the phenomenon is real. The conventional slip condition, Equation (2), evidently cannot predict this behavior; however, Maxwell's general slip condition produces just such a velocity. Close quantitative agreement between DSMC and simulation is not expected here, as the degree of gas rarefaction in this problem means that continuum fluid models are at the limit of their applicability.

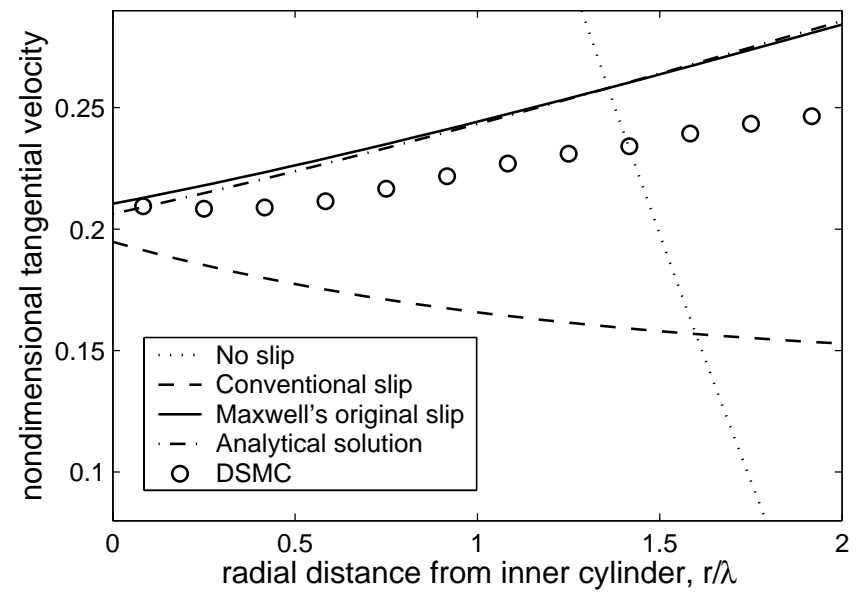

FIGURE 1. Nondimensional velocity profiles in simulations of cylindrical Couette flow. Comparison of no slip ( $\cdots)$, conventional slip (--) and Maxwell's general slip (-) solutions, an analytical solution [6] $(\cdot-\cdot)$ and DSMC data [7] (O).

\section{Drag on an unconfined sphere}

Isothermal slip flow past an unconfined sphere at very low Reynolds and Mach numbers was first analyzed by Basset [4] using Stokes' creeping flow approximation. The effect of slip was incorporated into the analysis using a velocity boundary condition for isothermal flows of an equivalent form to Maxwell's general boundary condition, Equation (1). Basset's analysis showed that the skin friction drag, $D_{s}$, on an unconfined sphere of radius $a$, in a flow stream of velocity $U$, can be written as:

$$
D_{s}=4 \pi \mu U a^{2}\left(\frac{\sigma}{\sigma(a-3 \lambda)+6 \lambda}\right)
$$

However, if the conventional form of Maxwell's boundary condition, Equation (2), is used in the derivation a different expression for the skin friction drag is obtained:

$$
D_{s}=4 \pi \mu U a\left(\frac{\sigma(a+\lambda)-2 \lambda}{\sigma(a-2 \lambda)+4 \lambda}\right)
$$

The disparity between the two drag predictions is due to the exclusion of curvature effects from the conventional boundary condition. In the limiting case of a perfectly smooth sphere, such that all incident molecules are reflected specularly $(\sigma=0)$, there is no means by which the wall can transfer tangential momentum to or from the gas. Therefore, the drag due to skin-friction should be zero and, indeed, we find that Basset's drag equation predicts no skin friction. However, Equation (5) predicts a finite value of negative skin friction drag (i.e. a thrust). This nonphysical prediction demonstrates the importance of employing the general, as opposed to the conventional, form of Maxwell's boundary condition for curved surfaces. 


\section{CONSTITUTIVE DEPENDENCE IN MAXWELL'S GENERAL FORMULATION}

While there is no commitment made to the form of the stress tensor or heat flux vector in Maxwell's general condition, Equation (1), normally the Navier-Stokes constitutive relations are assumed. This means that in flow situations where linear constitutive relations are no longer appropriate, the generality of the constitutive dependence in Maxwell's expression needs to be exploited.

For example, consider the DSMC simulation of a planar Poiseuille flow velocity profile, $U$, shown in Figure 2 [3]. The function ' $-A_{1} \lambda \tau / \mu$ ' (the dashed line) is proportional to the shear stress, and the function ' $A_{1} \lambda \mathrm{d} u / \mathrm{d} n$ ' (the dashed and dotted line) is proportional to the rate of strain. Towards the centre of the channel these functions converge, indicating that a Navier-Stokes model is appropriate for the "core" flow. Near the wall, however, the Knudsen layer represents a region of high local non-equilibrium in which the proportionality between stress and rate of strain breaks down. The conventional form of the slip boundary condition relates slip linearly to rate of strain so, in this case where the DSMC velocity profile is known, would predict a velocity slip (indicated by the triangle) almost three times greater than the actual slip. In contrast, the general form of Maxwell's condition provides an accurate prediction of the slip at the wall (indicated by the circle).

If the flow field is to be modeled throughout using the Navier-Stokes equations the two functions shown in Figure 2 are necessarily equal. However, when higher-order continuum methods are used, such as the Burnett equations [8], the simplified conditions of Equation (2) and Equation (3) can no longer be employed with confidence.

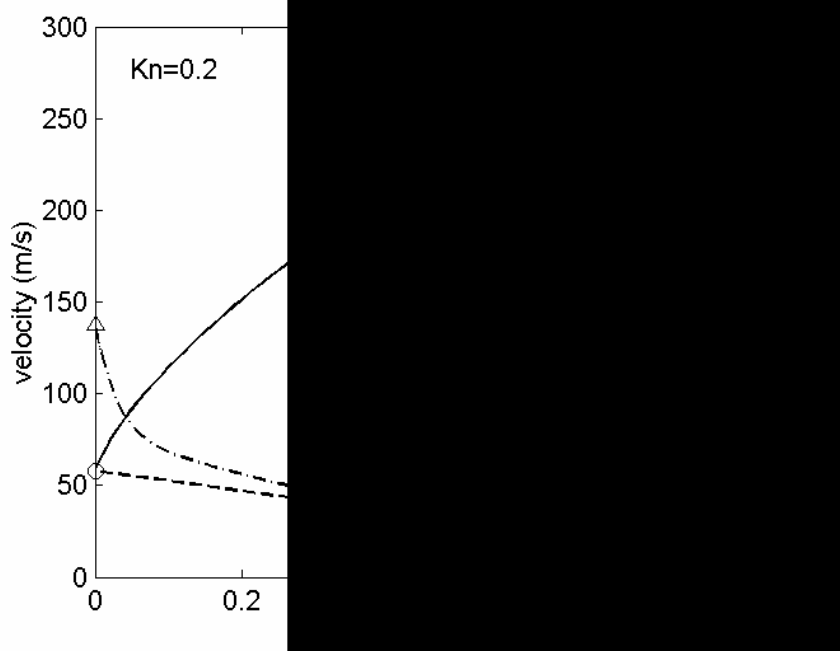

FIGURE 2. The velocity profile $U$ and derived quantities $-A_{1} \lambda \tau / \mu$ and $A_{1} \lambda \mathrm{d} u / \mathrm{d} n$ in a DSMC simulation of planar Poiseuille flow [3]. The gas is argon and the Knudsen number, based on half the channel width, is approximately 0.2 . The symbols $\Delta$ and o denote the slip predicted by Maxwell's conventional slip conditions, and the slip predicted by the general form, respectively. Here the slip coefficient has been taken as $A_{1}=\sqrt{2 / \pi}$.

\section{Thermal-stress slip flow}

Thermal-stress slip flow is a rarefaction phenomenon that was originally predicted by Sone [9]. Using an asymptotic analysis of the Boltzmann equation, he showed that a tangential variation in the wall-normal temperature gradient could induce velocity slip. Sone's configuration is a gas (initially stationary) between two stationary noncoaxial cylinders of different uniform temperature, $T_{1}$ and $T_{2}$. In the absence of thermal creep (i.e. the boundary temperature jump is not considered) no conventional boundary condition has the mechanism to predict a slip flow. Sone, however, calculated the slip-flow field as shown by the streamlines and directional arrows in Figure 3(a). The outer cylinder is held at a higher temperature and this generates a steady clockwise circulation in the gas (and anticlockwise when $T_{1}>T_{2}$ ).

For a similar cylindrical configuration, and using a finite volume code to solve the Burnett equations combined with Maxwell's general condition (1), we obtain the steady flow pattern shown in Figure 3(b). In the absence of 
thermal creep, no flow could be sustained by either of the slip conditions given in Equation (2) or Equation (3). It is therefore clear that the phenomenon predicted by Sone is captured in our simulation because of the inclusion of the general form of Maxwell's boundary condition.
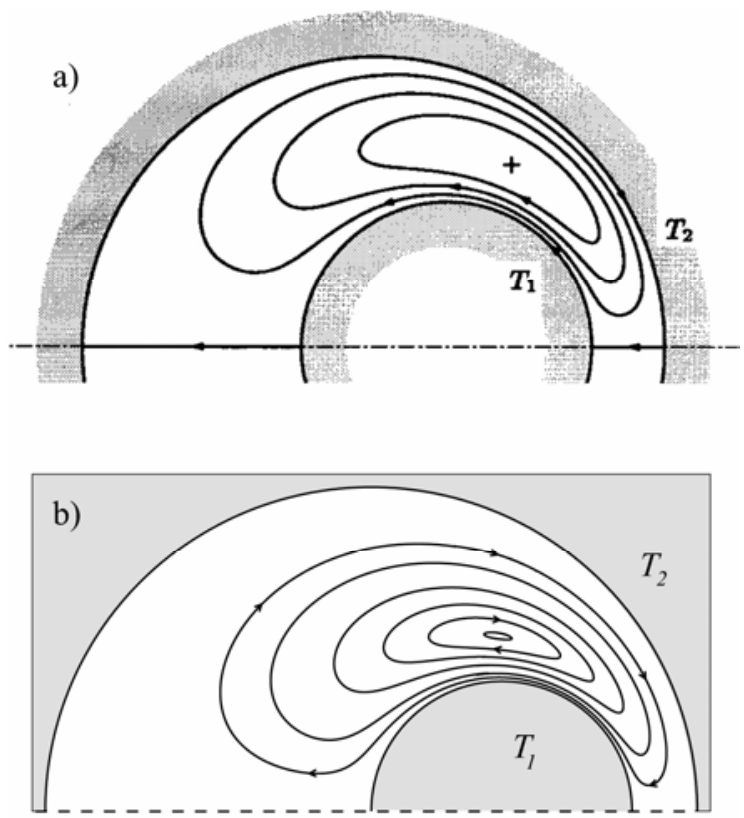

FIGURE 3. Streamlines of thermal-stress slip flow between non-coaxial cylinders (uniform temperatures, $T_{2}>T_{1}$ ); (a) solution of the Boltzmann equation reproduced from [10], (b) finite volume solution of the Burnett equations using the general form of Maxwell's boundary condition. Part (a) reprinted, with permission, from the Annual Review of Fluid Mechanics, Volume 32 (C)2000 by Annual Reviews www.annualreviews.org.

\section{NUMERICAL IMPLEMENTATION OF THE SLIP BOUNDARY CONDITION} by

For a Navier-Stokes simulation of a micro flow contained by a planar isothermal wall, the velocity slip is given

$$
u_{s}=\frac{(2-\sigma)}{\sigma} \lambda \frac{\mathrm{d} u_{x}}{\mathrm{~d} n}
$$

In this most basic configuration there is a physical requirement for the gradient of velocity to be of the same sign as the slip velocity. By using a finite difference approximation for the derivative, this can be interpreted as a numerical constraint:

$$
\frac{u_{s}}{u_{i}} \leq 1
$$

where $u_{i}$ is the streamwise velocity at the first internal grid point a distance $\Delta n$ away from the wall in the normal direction. When solved explicitly, Equation (6) is numerically unstable if the prediction of slip violates the condition given in Equation (7). This instability has been documented in [11] and places a serious restriction on the applicability of the boundary conditions. To demonstrate this we consider the discretized form of an explicit solution to Equation (6):

$$
u_{s}^{*}=\lambda \frac{u_{i}-u_{s}}{\Delta n}=\frac{\mathrm{Kn}}{\varepsilon}\left(u_{i}-u_{s}\right)
$$


where $u_{s}^{*}$ is the explicitly-predicted slip and $\varepsilon=\Delta n / L$ is a measure of the spatial resolution of the simulation (here we take $A_{1}=\sigma=1$ ). Assuming that the no-slip condition is the solution's starting point, Equation (7) can be combined with Equation (6) to give a condition for numerical stability:

$$
\varepsilon \geq \mathrm{Kn}
$$

In a Couette flow simulation with a resolution $\varepsilon=0.01$ (i.e. 100 grid points across the channel) the maximum stable Knudsen number would be approximately $\mathrm{Kn}=0.01$. For flows at the upper end of the slip-flow regime (up to $\mathrm{Kn}=0.1)$ no more than 10 grid points across the channel would be stable.

There are a number of ways to circumvent this problem (such as relaxation of the boundary conditions [12]), but the most efficient method is to treat the boundary condition implicitly. Equation (6) is rewritten implicitly, as follows:

$$
u_{s l i p}^{*}=\lambda \frac{u_{i}-u_{s l i p}^{*}}{\Delta n}=\frac{\lambda u_{i}}{\lambda+\Delta n}
$$

This form of the boundary condition is unconditionally stable. To show this, Equation (10) is combined with Equation (7) to give the following condition for stability:

$$
\varepsilon \geq 0
$$

which is always satisfied.

\section{SUMMARY}

In this paper we have demonstrated the importance of using the complete form of Maxwell's general boundary condition. Examples have been given where conventional and simplified versions of Maxwell's slip condition neglect the geometry- and constitutive-dependent characteristics of the general formulation and, as a result, fail to capture some important physical phenomena. We have also discussed a common numerical instability associated with the Maxwell slip condition and proposed a simple implicit solution technique to circumvent this problem.

\section{ACKNOWLEDGMENTS}

The authors are grateful to the UK's Leverhulme Trust (Research Project Grant No. F/07040/G) and Medical Research Council (MicroFAST programme, Grant No. 57719) for supporting this research financially.

\section{REFERENCES}

1. Maxwell, J. C., Phil. Trans. Roy. Soc. 170, 231-256 (1879).

2. Cercignani, C., Mathematical Methods in Kinetic Theory, Plenum Press, New York, 1990, pp. 232-243.

3. Lockerby, D. A., Reese, J. M., and Gallis, M. A., "A wall-function approach to incorporating Knudsen-layer effects in gas micro flow simulations" in Proceedings of the $24^{\text {th }}$ International Symposium on Rarefied Gas Dynamics, Bari, Italy (2004).

4. Basset, A. B., A Treatise on Hydrodynamics, Cambridge University Press, Cambridge, 1888

5. Aoki, K., Yoshida, H., Nakanishi, T., and Garcia, A. L., Phys. Rev. E 68, 016302 (2003).

6. Einzel, D., Panzer, P., and Liu, M., Phys. Rev. Lett. 64, 2269-2272 (1990).

7. Tibbs, K. W., Baras, F., and Garcia, A. L., Phys. Rev. E 56, $2282-2283$ (1997).

8. Reese, J. M., Gallis, M. A., and Lockerby, D. A., Phil. Trans. Roy. Soc. 361, 2967-2988 (2003).

9. Sone, Y., Phys. Fluids 15, 1418-1423 (1972).

10. Sone, Y., Ann. Rev. Fluid Mech. 32, 779-811 (2000).

11. Karniadakis, G. E., and Beskok, A., Micro Flows: Fundamentals and Simulation, Springer, Berlin, 2002.

12. Lockerby, D. A., and Reese, J. M., J. Comput. Phys. 188, 333-347 (2003). 